[gw22-e0605] INSULIN TREATMENT EXERTS

\title{
CARDIOPROTECTIVE EFFECT AGAINST REPERFUSION INJURY VIA INHIBITING EARLY REPERFUSION-INDUCED CAMKII ACTIVATION
}

Yan Wenjun', Xing Wenjuan², Liu Peilin', Zhang Haifeng², Wang Haichang', Tao Ling', Gao Feng ${ }^{3}$ Department Of Cardiology, Xijing Hospital, Xijing, China; ${ }^{2}$ Department Of Physiology, Fourth Military Medical University; ${ }^{3}$ Department Of Physiology And Department Of Cardiology, Xijing Hospital, Xijing, China

10.1136/heartjnl-2011-300867.215

Objectives Primary reperfusion therapies, including percutaneous coronary intervention (PCI) and thrombolysis, are the standard of care for treating myocardial infarction (MI). Paradoxically, however, the return of blood flow can also result in additional damage and complications, referred to as reperfusion injury. In ischemic/reperfused (I/R) heart, Ca2+/calmodulin-dependent protein kinase II (CaMKII) activation has been implicated in triggering apoptotic signaling. We have previously found that insulin reduces I/R injury, but the mechanisms remain elusive. The present study was designed to determine whether early insulin treatment reduces the myocardial reperfusion injury at the onset of $\mathrm{R}$ and its mechanisms.

Methods and Results Male adult SD rats were subjected to $30 \mathrm{~min}$ of MI followed by $3 \mathrm{~h}$ of R. A significant activation of CaMKII at 3 min of $\mathrm{R}$ was confirmed by increased phosphorylation of both CaMKII (1.4-fold) and PLBThr17 (9.7-fold). Insulin treatment $(0.4 \mathrm{U} / \mathrm{kg} / \mathrm{h}$, administrated $10 \mathrm{~min}$ before MI and during the first $10 \mathrm{~min}$ of $\mathrm{R}$, intravenous) notably improved PI3K expression and pAkt/Akt ratio ( $p<0.01$ vs Saline) and inhibited CaMKII activity at 3 min of R (pCaMKII/CaMKII, pPLBThr17/PLB, both $p<0.01$ ). At the end of $3 \mathrm{~h}$ of $R$, insulin group exhibited reduced I/R injury as evidenced by decreased apoptosis (TUNEL and activated caspase 3 ) and improved left ventricular developed pressure recovery (LVDP: $77.2 \% \pm 2.9 \%$ vs $65.3 \% \pm 2.5 \%$, all $\mathrm{p}<0.05)$. Pretreatment with wortmannin partially abolished insulin's inhibition of CaMKII activity $(\mathrm{p}<0.01)$ and its reduction of reperfusion injury (decreased TUNEL, activated caspase 3 and increased LVDP, all $\mathrm{p}<0.05)$. 
Conclusion These data are the first to demonstrate that insulin inhibits CaMKII in the beginning of reperfusion, at least in part, through PI3K-Aktsignaling and reduces reperfusion injury, which suggests that early insulin therapy when reperfusion is clinically performed exerts significant cardioprotection. 